\title{
Labor Adjustment Costs and Complex Eigenvalues
}

\author{
Xavier Fairise \\ University of Evry (EPEE) \\ Patrick Fève* \\ University of Toulouse (GREMAQ-CNRS and IDEI) \\ and Banque de France
}

\begin{abstract}
Aggregate fluctuations display both persistence and damped oscillations in response to transitory shocks. The standard Real Business Cycle model cannot explain these patterns, because its stable eigenvalues are positive and real. We demonstrate that this model with labor adjustment costs can yield complex eigenvalues. However, numerical experiments suggest that the model cannot display distinguishable damped oscillations of aggregate variables.
\end{abstract}

Keywords : Labor adjustment costs, Business cycle model, Complex eigenvalues.

J.E.L. classification : C12, C52

*Address: Gremaq, Université de Toulouse I, Manufacture des Tabacs, Bâtiment F, 2ème étage, 21 Allée de Brienne, 31000 Toulouse, France. e-mail: patrick.feve@univ-tlse1.fr. We thank P. Beaudry, J.P. Benassy, F. Collard, P. Flaschel, P.Y. Hénin, T. Kollintzas, F. Langot, F. Portier, W. Zemmler, an associate editor and an anonymous referee for fruitful remarks and comments. An earlier version of this paper has benefited from discussions at the workshop Human Capital Mobility (Louvain-la-Neuve, sept. 1996), T2M Conference (Montreal, may 1999), SED conference (Alghero ,june 1999), ESEM99 (Santiago, august 1999), GEAPE seminar (Angers, december 1999) and “Microstructures, Macrodynamics and Macroeconometrics” workshop (Bielefeld, january 2000). The usual disclaimers apply. 


\section{Introduction}

Aggregate output fluctuations and related measures of economic activity display both persistence and damped oscillations in response to transitory shocks. For Azariadis, Bullard and Ohanian [2001], this pattern appears to be a robust empirical finding through both the roots of simple autoregressive and vector autoregressive representations of aggregate variables. The standard Real Business Cycle (RBC) model, in the sense of the one sector optimal growth model governed by a technological shock, cannot explain these stylized facts. This failure of the standard RBC model partly results in its inability to display persistent damped oscillations, These oscillations are characterized in any dynamic model by complex eigenvalues with large imaginary part compared to the real one. As pointed out by Azariadis et al. [2001], building models in accordance with these business cycle facts is actually sensible.

This paper shows that the standard RBC model with a slight modification is qualitatively able to produce complex eigenvalues. The extension concerns the labor input, which is now considered as a quasi-fixed factor. The standard model abstracts from employment lags. But, as suggested by Oi [1962], labor displays smooth adjustments along the business cycle, usually modelled by labor adjustment costs. For small costs, the model behaves as a standard RBC model. Conversely, large costs imply a labor almost constant over time. Complex eigenvalues occur if changes in labor input are costly and the intertemporal substitution of consumption is sufficiently large. Labor adjustment costs implies persistent deviations of employment, whereas high intertemporal substitution of consumption induces high sensitivity of saving to change in the real interest rate. Following a positive shock that increases employment, the labor input will go back slowly to its steady state as it is costly to adjust. As capital will gradually increases, the real interest rate remains above its long run value. After some periods, the increase in capital implies that the real interest rate will be below its long run value. When intertemporal substitution of consumption is sufficiently large, household will reduce strongly their saving and the real interest rate will increase again and move above its long run value. Aggregate variables can thus fluctuate around the steady state.

The paper also shows that sufficiently conditions for complex eigenvalues are satisfied for most preferences specifications typically used in the RBC literature. Some numerical experiments illustrate this property but they suggest that the imaginary part remains insufficiently large compared to the real one. This means that the response of aggregate variables cannot display any distinguish- 
able oscillations when they go back to their steady state values. Moreover, we show that the RBC model with labor adjustment cost can display persistence. Nevertheless, we question the empirical relevance of the model, as it implies an excess smoothness of employment.

The paper is organized as follows. A first section presents the model economy. Section 2 characterizes the local dynamic properties of the model and discusses the conditions under which complex eigenvalues occur. Section 3 presents some numerical experiments. A last section offers some concluding remarks. Proofs are given in appendix.

\section{The model}

There exists a single good both consumed and invested. The economy is populated by an infinite number of identical agents with infinite lifetime. Their preferences are described by a time separable utility function in consumption and leisure $u\left(C_{t}, L_{t}\right)$. Time endowment is normalized to one and hours worked are given by $N_{t}=1-L_{t}$. The utility function satisfies the following conditions:

Assumption: (i) The utility function $\left.u():. \mathbb{R}_{+}^{*} \times\right] 0,1\left[\rightarrow \mathbb{R}_{+}^{*}\right.$ is strictly increasing and concave in $C$ and $L \equiv 1-N$, (ii) verifies the additional restrictions $u_{C L} u_{L}-u_{L L} u_{C} \geq 0$ and $u_{C L} u_{C}-$ $u_{C C} u_{L} \geq 0$ with at least one strict inequality and (iii) satisfies the Inada conditions.

Condition $(i)$ is rather standard, whereas condition (ii) imposes that consumption and leisure are normal goods. We will see later that this restriction is central for the saddle path property. Because the approximate solution is obtained through a log-linearization around the steady state, it is useful to express previous conditions in terms of elasticities of the marginal utilities:

$$
\xi_{C C}=C u_{C C} / u_{C} \quad \xi_{C L}=L u_{C L} / u_{C} \quad \xi_{L C}=C u_{C L} / u_{L} \quad \xi_{L L}=L u_{L L} / u_{L}
$$

Using these elasticities, the condition (i) becomes $\xi_{C C} \xi_{L L}-\xi_{C L} \xi_{L C} \geq 0$ and (ii) $\xi_{C L}-\xi_{L L} \geq 0$ and $\xi_{L C}-\xi_{C C} \geq 0$ with at least one strict inequality. The condition (iii) also insures the existence and uniqueness of the steady state.

The technology is described by a Cobb-Douglas production function with constant returns to scale

$$
\bar{Y}_{t}=Z K_{t}^{1-\alpha} N_{t}^{\alpha}
$$

with $0<\alpha<1 . K_{t}, N_{t}, \bar{Y}_{t}$ and $Z>0$ denote the capital stock, the labor input, the raw product and the level of the technology, respectively. Capital accumulation is described by the following 
law of motion

$$
K_{t+1}=(1-\delta) K_{t}+I_{t}
$$

where $\delta \in] 0,1\left[\right.$ denotes the depreciation rate and $I_{t}$ is the flow of investments. The employment evolves according to

$$
N_{t+1}=(1-\nu) N_{t}+H_{t}
$$

where $\nu \in] 0,1\left[\right.$ is the quit rate and $H_{t}$ represents the flow of hirings. Productive employment at time $t+1$ is hired at time $t$, implying some labor hoarding phenomenon (see Burnside, Eichenbaum and Rebelo [1993] and Fairise and Langot [1994] $]^{1}$ ). Labor is a quasi-fixed factor. The adjustment costs function follows a standard quadratic specification:

$$
\mathcal{G}\left(H_{t}, N_{t}\right)=\frac{b}{2} \frac{\left(H_{t}-\nu N_{t}\right)^{2}}{N_{t}}
$$

with $b>0$. This function satisfies convexity and is homogeneous of degree one. The decision rule on hirings is thus independent of the size of the economy and the hiring rate only depends on the marginal value of labor. At the steady state, this function satisfies $\mathcal{G}()=.\mathcal{G}_{H}()=.\mathcal{G}_{N}()=$. and $\mathcal{G}_{H H}()=.b / N^{*}$, where $N^{*}$ denotes the steady state employment. This implies that the steady state of the model does not differ from the one of the standard model. Adjustment costs only affect the convergence path toward the steady state. This allows us to concentrate on the dynamic implications of labor adjustment costs.

The aggregate resources constraint is given by :

$$
Z F\left(K_{t}, N_{t}\right)-\mathcal{G}\left(H_{t}, N_{t}\right)=C_{t}+I_{t}
$$

The central planer solves the following intertemporal problem :

$$
\max _{I_{t}, H_{t}} \sum_{i=0}^{\infty} \beta^{i} u\left(C_{t+i}, 1-N_{t+i}\right)
$$

subject to the period-by-period aggregate resources constraint (4), the laws of motion on capital (2) and employment (3) and for $K_{0}, N_{0}$ given and strictly positive. The parameter $\left.\beta \in\right] 0,1[$ denotes the constant discount factor. The first order conditions are:

$$
p_{t}=u_{C}(t)
$$

\footnotetext{
${ }^{1}$ Note that our model departs from Burnside et al. [1993] and Fairise and Langot [1994], as we do not introduce variable intensity of work effort. In our model, firms can not adjust their inputs, whereas firms can adjust the intensive margin in Burnside et al. [1993] and Fairise and Langot [1994]
} 


$$
\begin{aligned}
\lambda_{t} & =u_{C}(t) \mathcal{G}_{H}(t) \\
p_{t} & =\beta\left\{u_{C}(t+1) Z F_{K}(t+1)+(1-\delta) p_{t+1}\right\} \\
\lambda_{t} & =\beta\left\{u_{C}(t+1)\left(Z F_{N}(t+1)-\mathcal{G}_{N}(t+1)\right)-u_{L}(t+1)+(1-\nu) \lambda_{t+1}\right\}
\end{aligned}
$$

where $p_{t}$ and $\lambda_{t}$ are the implicit prices of capital and labor, respectively. These two implicit prices satisfy usual terminal conditions. The first order conditions (5)-(8), the aggregate resources constraint (4) and the laws of motion (2) and (3) define the optimal path of the economy.

\section{Dynamic properties}

This section establishes the dynamic properties of the model. We report in appendix A the linearized model, its transformation and some general results. ${ }^{2}$ With our specification of the labor adjustment costs, the steady state corresponds exactly to the one of the standard RBC model. There exists an unique steady state $\left(I^{\star}, K^{\star}, H^{\star}, N^{\star}, p^{\star}, \lambda^{\star}, C^{\star}\right)$ that satisfies: $I^{\star}-\delta K^{\star}=0, H^{\star}-\nu N^{\star}=$ $0, \beta\left[Z F_{K}\left(K^{\star}, N^{\star}\right)+1-\delta\right]-1=0, u_{C}\left(C^{\star}, 1-N^{\star}\right) Z F_{N}\left(K^{\star}, N^{\star}\right)-u_{L}\left(C^{\star}, 1-N^{\star}\right)=0$, $p^{\star}=u_{C}\left(C^{\star}, 1-N^{\star}\right), \lambda^{\star}=0$ and $Z F\left(K^{\star}, N^{\star}\right)-C^{\star}-I^{\star}=0$. Given these steady state values, we then study the dynamic properties of the log-linear version of (2)-(8). We first establish the following property:

Proposition 1 If the assumptions (i) and (ii) on the utility function hold, then there exists a unique convergence path toward the steady state.

Proposition 1 shows that the introduction of labor adjustment costs does not modify the saddle path property of the standard RBC model. Note that our assumptions on the utility function, i.e. consumption and leisure are normal goods, are sufficient to establish this result. Compared to the standard RBC model, we only add an additional restriction that insures the saddle path property, that is the convexity of the adjustment costs function $(b>0)$. Given this result, we now study in details other interesting dynamic properties of the model. The following proposition raises the possibility for complex eigenvalues.

Proposition 2 If the preferences satisfy the conditions :

$$
\begin{aligned}
\xi_{C C} & \geq-1 \\
\xi_{C C}-\xi_{L C} & \leq-1
\end{aligned}
$$

\footnotetext{
${ }^{2}$ More details are avialable from the authors upon request.
} 
then, there exists an interval $[\underline{b}, \bar{b}]$, with $0<\underline{b}<\bar{b}<\infty$, such that eigenvalues are (i) complex if $b \in] \underline{b}, \bar{b}[$ and (ii) real if $b \in] 0, \underline{b}] \cup[\bar{b},+\infty[$.

The existence of a complex eigenvalues imposes some restrictions on preferences. The elasticity $\xi_{C C}$ appears in both conditions. This shows that the specification of the utility function matters for the dynamic property of the model economy. Conversely, none of the structural parameters that characterize the technology and the accumulation process enters in the sufficient conditions.

The intuition of such a dynamic property is the following. Suppose that a positive shock hits employment above its steady state value. When labor adjustment costs are zero, the economy will go back quickly to its steady state as changes in labor input are costless. Conversely, when these changes are costly, employment will go back slowly to its steady state. As capital will gradually adjust, the real interest rate increases. After some periods, the increase in capital will critically lower the real interest rate below its long run value. When intertemporal substitution effect in consumption is sufficiently large, household will have incentives to reduce saving. This decrease in saving will create an upward pressure on the real interest rate. The real interest rate (and other aggregate variables) can thus fluctuate around its steady state value.

Few parameters enter in the sufficient conditions (9) and (10). It follows that complex eigenvalues can therefore be easily checked. The following examples illustrates the proposition.

Example 1 Consider the isoelastic utility function:

$$
u\left(C_{t}, 1-N_{t}\right)=\frac{1}{1-\sigma}\left[C_{t}^{\theta}\left(1-N_{t}\right)^{1-\theta}\right]^{1-\sigma}
$$

with $\theta \in] 0,1[$ and $\sigma \in] 0,1[\cup] 1, \infty[$. It is for instance the one used by Kydland and Prescott [1982]. We have $\xi_{C C}=\theta(1-\sigma)-1$ and $\xi_{C C}-\xi_{L C}=-1$. Condition (10) is always satisfied and condition (9) hold if $\sigma \leq 1$. The standard case of logarithmic and separable utility function satisfies these conditions. In this case, $\sigma=1, \xi_{C C}=-1$ and $\xi_{L C}=0$.

Example 2 Consider the utility function with indivisible labor supply proposed by Hansen [1985] and Rogerson [1988]:

$$
u\left(C_{t}, 1-N_{t}\right)=\log \left(C_{t}\right)+\theta\left(1-N_{t}\right)
$$


We directly deduce that $\xi_{C C}=-1$ and $\xi_{C C}-\xi_{L C}=-1$ and conditions (9) and (10) are satisfied.

Example 3 Consider the class of utility functions that produces static labor supply:

$$
\log \left(C_{t}-\psi_{0} \frac{N_{t}^{1+\psi}}{1+\psi}\right)
$$

with $\psi, \psi_{0}>0$. This function, used by Hercowitz and Sampson [1991] among others, implies that the income effect on leisure is zero. It follows that $\xi_{C L}-\xi_{L L}=\psi L^{*} /\left(1-L^{*}\right)>0$ and $\xi_{C C}-\xi_{L C}=0$. The condition (10) is thus not verified.

In example 1, the condition (9) is not verified if $\sigma>1$. Nevertheless, a less restrictive condition can be obtained from the very plausible assumption that the labor share exceeds the depreciation rate of the capital.

Proposition 3 If the preferences satisfy the conditions :

$$
\begin{aligned}
& \xi_{C C} \geq-(1+\alpha) \\
& \xi_{C C}-\xi_{L C} \leq-1
\end{aligned}
$$

and if $\alpha>\delta$, then, there exists an interval $[\underline{b}, \bar{b}]$, with $0<\underline{b}<\bar{b}<\infty$, such that the eigenvalues are (i) complex if $b \in] \underline{b}, \bar{b}[$ and (ii) real if $b \in] 0, \underline{b}] \cup[\bar{b},+\infty[$.

We immediately see that the condition (11) is less restrictive than the condition (9). In example 1 , when $\sigma=1.5, \theta=1 / 3, \alpha=0.64$ and $\delta=0.025$ as in Kydland and Prescott [1982], complex eigenvalues can occur. The condition (12) in proposition 3 is exactly the same than condition (10) in proposition 2. It follows that example 3 does not verify condition (12).

\section{Numerical experiments}

\subsection{Sensitivity Analysis}

Following example 2, we choose a utility function with indivisible labor supply. ${ }^{3}$ From our assumptions on the structure of the labor adjustment costs, the steady state of the model is the same than the one of the standard RBC model. This allows to set the values of the structural parameters

\footnotetext{
${ }^{3}$ A similar exercice have been performed with isoelastic utility function. The results are quite similar, despite a lower imaginary part of the eigenvalues.
} 
in accordance to previous calibrations and thus to use freely the parameter $b$ of labor adjustment costs.

Table 1: Values of the structural parameters

\begin{tabular}{cccc}
\hline \hline Technology & \multicolumn{3}{c}{ Preferences } \\
\hline$\alpha$ & 0.640 & $\beta$ & 0.99 \\
$\delta$ & 0.025 & $N^{\star}$ & 0.40 \\
$\nu$ & 0.015 & & \\
\hline \hline
\end{tabular}

The parameter $\alpha$ corresponds to a labor share of $64 \%$ at steady state. The parameter $\beta$ is set in order to imply a $4 \%$ annual subjective discount rate. The depreciation rate $\delta$ is equal to $2.5 \%$ per quarter. The quit rate $\nu$ is fixed in order to roughly match the average destruction rate in the US manufacturing sector over the period $1972-1993 .{ }^{4}$ The time spent to productive activity is equal to $40 \%$. The value of $\theta$ is thus deduced from the steady state conditions. Finally, the parameter of the production function $Z$ is set to scale the adjustment costs parameter. So, in what follows, the value of $b$ must be interpreted with respect to the scale parameter $Z$. All these values are reported in table 1.

Figure 1 presents the modulus, the real part and the imaginary part of the two eigenvalues with respect to the adjustment costs parameter $b$. For $b$ small, the two eigenvalues are real. As $b$ increases, the modulus of these two eigenvalues becomes closer and then complex conjugate. However, for $b$ large (not reported in figure 1), the imaginary part is zero. A disappointed quantitative result concerns the size of the imaginary part, as it remains insufficiently large - it never exceeds 0.025 - compared to the real part - it is close to 0.95-. This result suggests the model cannot generate damped oscillations in response to transitory shocks, i.e. the response of aggregate variables does not display any distinguishable oscillations when they go back to their steady state values.

We further explore the quantitative effects of other structural parameters changes on aggregate dynamics. We compute the imaginary part of the eigenvalue with respect to the adjustment costs parameter $b$ and a selected structural parameter. We keep a utility function linear in leisure, but we consider that the elasticity $\xi_{C C}$ can differ from minus unity. The four structural parameters are the steady state labor share $\alpha$, the depreciation rate $\delta$, the discount factor $\beta$ and the curvature of the

\footnotetext{
${ }^{4}$ If $N_{t}$ should be interpreted as hours rather than employment, the calibration of $\nu$ should be adjusted accordingly. Nevertheless, our numerical results has appeared unsensitive to various values of this parameter.
} 
Figure 1: Roots with labor adjustment costs
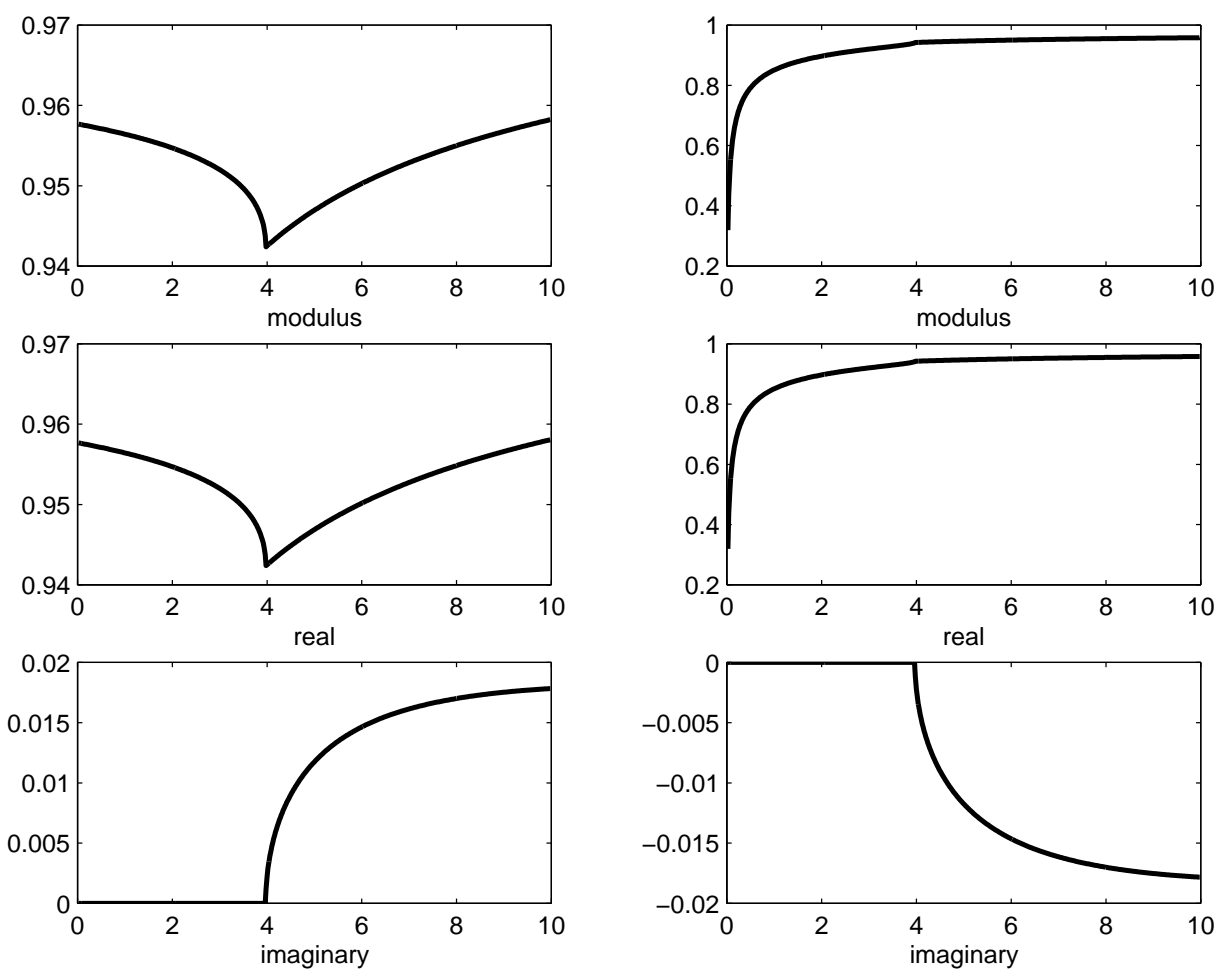

Figure 2: Imaginary part
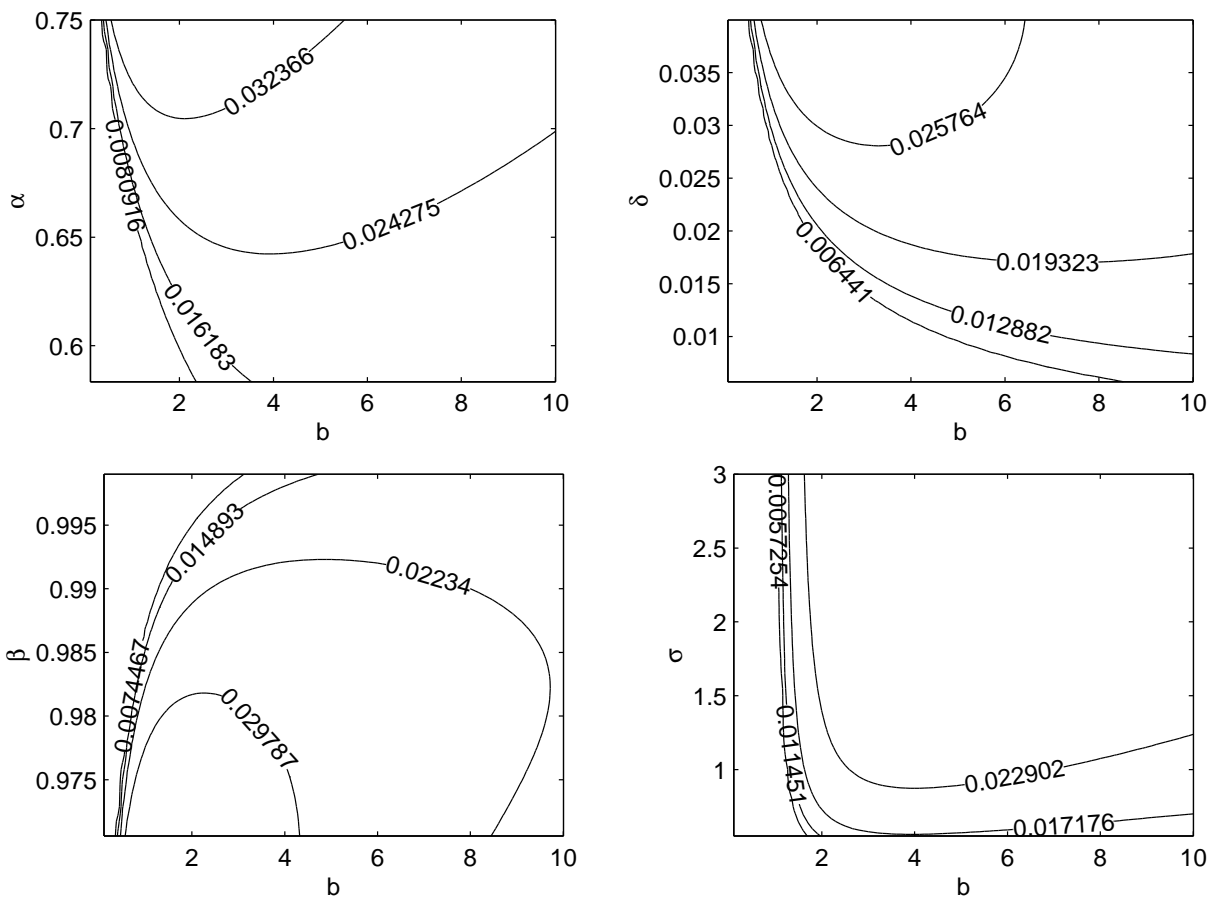
utility function with respect to consumption $\sigma=-\xi_{C C}$. The range for $\alpha \in[0.58 ; 0.75]$ reflects on how proprietors' income is treated, i.e. the share of total output paid to capital varies between 0.25 and 0.42 . The range for $\delta \in[0.005 ; 0.040]$ is selected because it is commonly set to 0.025 and previous estimates lie within the selected range. The range for $\beta \in[0.970 ; 0.999]$ implies the annual subjective discount rate lies within [0.4\%;10.3\%]. Finally, the range for the curvature of the utility function $[0.5 ; 3]$ roughly corresponds to previous estimates. In each case, one of the structural parameter varies within the range, whereas the others are fixed to their reference values (see table 1). We report in figure 2 the contours of the 3-D function that express the imaginary part of the eigenvalue as a function of $b$ and $\{\alpha, \delta, \beta, \sigma\}$. The results, reported in Figure 2, are again disappointed as the imaginary part remains too small compared to the real part. Indeed, the real part in these experiments (not reported here) always exceeds 0.95, whereas the imaginary part never exceeds 0.035 .

\subsection{Transitional Dynamics}

The previous quantitative experiments suggest that the model cannot produce damped oscillations. Nevertheless, labor adjustment costs allows to generate persistent responses of aggregate variables. We report in figure 3 the transitional dynamics of capital, labor, consumption and investment when employment is above its steady state value. We consider two cases. In the first one, labor costs are zero $(b=0)$. In the second one, the adjustment cost parameter $b$ is chosen with the interval $[\underline{b}, \bar{b}]$. When labor adjustment costs are zero, employment quickly goes back to its long run value. It follows that the economy does not display any persistence. Conversely, when changes in labor input are costly, the labor input will adjust slowly. Employment is thus persistently above its steady state. These dynamic properties of employment will affect all the other aggregate variables. The response of capital stock and consumption is hump-shaped.

This experiment suggests that labor adjustment cost can improve the dynamic properties of the RBC model. It is worth noting that we conduct this experiment when the model exhibits complex eigenvalues. Nevertheless, as already mentioned, figure 3 illustrates the lack of significant oscillations. 
Figure 3: Transitional Dynamics
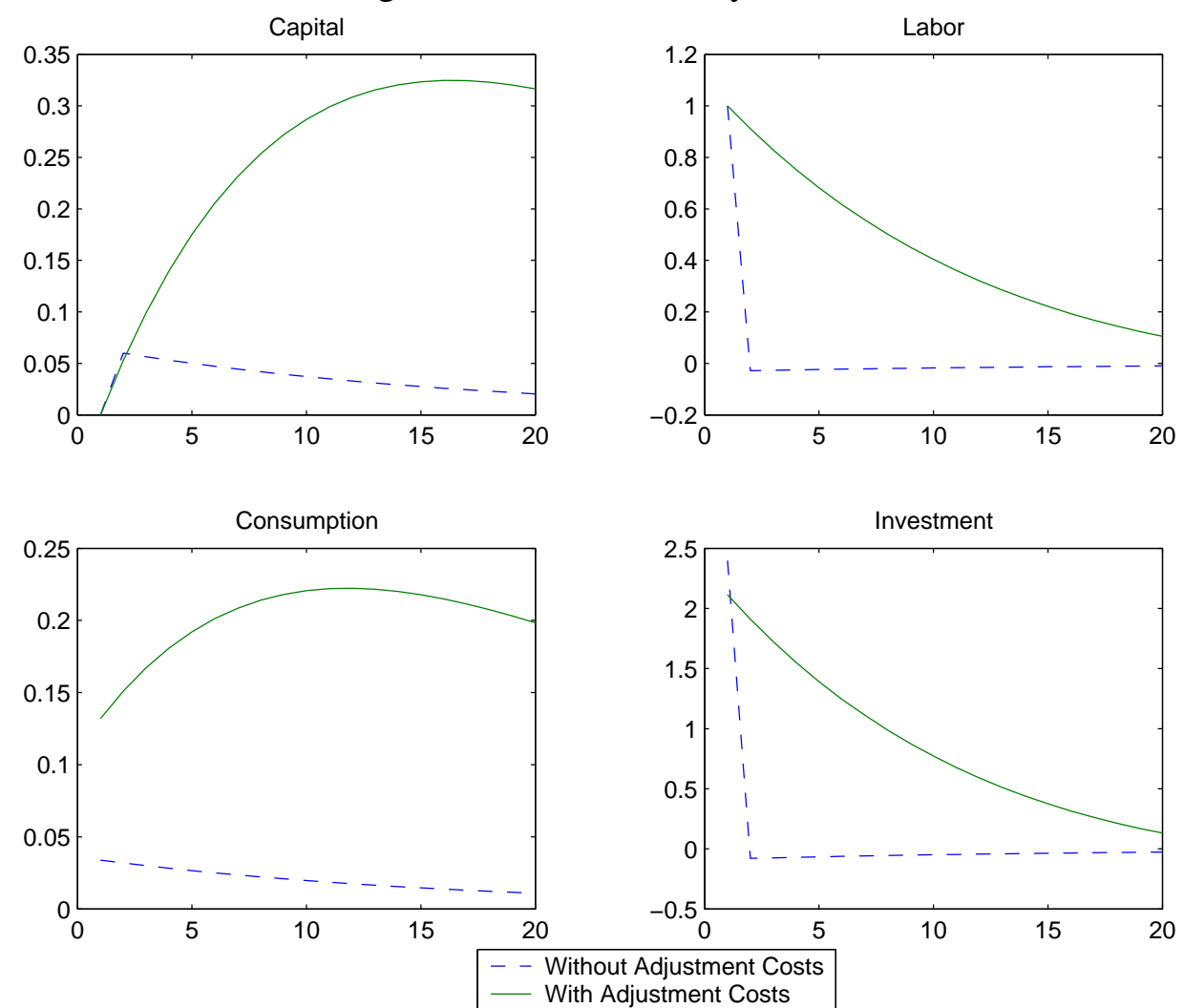

\subsection{Business Cycle Properties}

The previous experiments suggest that labor adjustment costs allows to generate persistent fluctuations in the RBC model. However, large labor adjustment costs tend to smooth the response of employment and thus other aggregate variables. We now inspect the business cycle properties of the model. We consider again two cases: $b=0$ and $b \in[\underline{b}, \bar{b}]$. We simulate the model when the economy is only perturbed by a stationary technology shock. As usual, the autoregressive parameter of this shock is equal to 0.95 . The standard error of the innovation is set in order to match the volatility of the cyclical component of the US Gross Domestic Product. ${ }^{5}$ We then inspect the model's business cycle properties regarding other aggregate variables. In table 2, we report various moments on these variables. Moments on US data come from King and Rebelo [1999]. The columns "Model (1)" and "Model (2)" report the business cycle properties of the RBC model without and with labor adjustment costs, respectively.

The relative volatility of consumption $\left(\sigma_{c} / \sigma_{y}\right)$ and investment $\left(\sigma_{i} / \sigma_{y}\right)$ are very similar in the two models. Note that they implies both an excess smoothness of consumption. The main departure

\footnotetext{
${ }^{5}$ This cyclical component is obtained from the Hodrick-Prescott filter.
} 
Table 2: Selected Moments

\begin{tabular}{lccc}
\hline \hline & US Data & Model (1) & Model (2) \\
\hline$\sigma_{y}$ & 1.81 & 1.81 & 1.81 \\
$\sigma_{c} / \sigma_{y}$ & 0.74 & 0.28 & 0.33 \\
$\sigma_{i} / \sigma_{y}$ & 2.93 & 3.25 & 3.03 \\
$\sigma_{n} / \sigma_{y}$ & 0.99 & 0.84 & 0.19 \\
$\rho_{n}$ & 0.88 & 0.68 & 0.93 \\
\hline \hline
\end{tabular}

Note: US Data: 1947.1-1996.4 (see King and Rebelo [1999]); Model (1): without adjustment costs; Model (2): with adjustment costs.

between the two models concerns the volatility of employment $\left(\sigma_{n} / \sigma_{y}\right)$. The model without adjustment cost implies a volatility of labor input close (but lower) to the one of the US data. Conversely, labor adjustment costs dramatically reduces the volatility of employment. This constitutes the second disappointed result of our model. However, labor adjustment costs improve the ability of the model to replicate the observed persistence of employment, i.e. the first order serial correlation of labor $\left(\rho_{n}\right)$. These two features illustrate the trade-off between an excess smoothness of employment and its persistence. This point has been already stressed by Cogley and Nason [1995]. They shown that a RBC model with labor adjustment costs account for serial correlation, but it fails to replicate observed impulse-response functions to a technology shock.

\section{Concluding remarks}

This paper studies the ability of a standard RBC model with labor adjustment costs to produce complex eigenvalues. The paper establishes sufficient conditions for complex eigenvalues and illustrates these properties using numerical experiments. However, the paper shows that labor adjustment costs can not display distinguishable aggregate oscillations and imply excess smoothness of employment. Further research must therefore explore the dynamic and quantitative properties of equilibrium models when labor adjustment costs are combined with suitable assumptions on good and labor market arrangements. 


\section{Appendix}

\section{A Notations and the log-linearized model}

This appendix derives the main dynamic properties of our model economy. We first introduce some notations: (1) elasticities of the adjustment cost functions: $\omega_{H H}=H^{*} \mathcal{G}_{H H} / Z F_{N}, \omega_{H N}=N^{*} \mathcal{G}_{H N} / Z F_{N}$, $\omega_{N H}=H^{*} \mathcal{G}_{N H} / Z F_{N}$ and $\omega_{N N}=N^{*} \mathcal{G}_{N N} / Z F_{N}$ with $N \mathcal{G}_{H N}+H \mathcal{G}_{H H}=0$; (2) elasticities of the marginal utilities : $\xi_{C C}=C^{*} u_{C C} / u_{C}, \xi_{C L}=L^{*} u_{C L} / u_{C}, \xi_{L C}=C^{*} u_{C L} / u_{L}$ and $\xi_{L L}=L^{*} u_{L L} / u_{L}$; (3) elasticity of the marginal product of capital $\eta_{K}=-\alpha(1-\beta(1-\delta))$; (4) consumption share $s_{C}=C^{*} / Y^{*}=$ $(1-\beta(1-\alpha \delta))(1-\beta(1-\delta))^{-1} ;(5)$ investment share $s_{I} \equiv 1-s_{c}=((1-\alpha) \delta \beta)(1-\beta(1-\delta))^{-1} ;(6)$ others: $\phi=1 / \delta, \psi=1 / \nu$. Let $x$ denotes the state variables $(K, N)$. After some algebra, the log-linearized dynamical system formed by (2)-(8) takes the following form :

$$
\Delta \widehat{x}_{t+2}+\Gamma \widehat{x}_{t+1}+\beta^{-1} \Delta^{\prime} \widehat{x}_{t}=0
$$

where the elements of the matrices $\Delta$ and $\Gamma$ are:

$$
\begin{array}{r}
\delta_{11}=\frac{K^{*}}{\beta}\left[-\frac{\phi s_{I}}{s_{C}} \xi_{C C}\right] \delta_{12}=0 \quad \delta_{21}=\frac{K^{*}}{\beta}\left[-\beta \frac{\alpha}{s_{C}}\left(\xi_{C C}-\xi_{L C}\right)\right] \delta_{22}=\frac{K^{*}}{\beta} \frac{\alpha \beta}{\phi_{s I}}\left[\psi \omega_{H H}\right] \\
\gamma_{11}=\frac{K^{*}}{\beta}\left[\eta_{K}+\left(1+\frac{1}{\beta}\right) \frac{\phi s_{I}}{s_{C}} \xi_{C C}\right] \gamma_{12}=\frac{K^{*}}{\beta}\left[-\eta_{K}+\frac{\alpha}{s_{C}}\left(\xi_{C C}-\xi_{L C}\right)\right] \\
\gamma_{22}=\frac{K^{*}}{\beta}\left[\frac{\alpha \beta}{\phi s_{I}}\left(-\psi \omega_{H H}-\frac{1}{\beta} \psi \omega_{H H}\right)+\eta_{K}+\frac{\alpha \beta}{\phi s_{I}}\left(\frac{\alpha}{s_{C}}\left(\xi_{C C}-\xi_{L C}\right)+\frac{N^{*}}{1-N^{*}}\left(\xi_{L L}-\xi_{C L}\right)\right)\right]
\end{array}
$$

For practical reasons, we transform equation (A.1) in a canonical form by the mean of a diagonalization. We follow an idea of Magill [1979] adapted by Cassing and Kollintzas [1991] to the case of a discrete time model. Such a method allows to highlight the symmetric and asymmetric characteristics of the dynamic system. We define the variable $\widehat{w}_{t}$ such that $\widehat{x}_{t}=\left(\beta^{-1 / 2}\right)^{t} \widehat{w}_{t}$ and (A.1) becomes :

$$
\Delta \widehat{w}_{t+2}+\Gamma \beta^{1 / 2} \widehat{w}_{t+1}+\Delta^{\prime} \widehat{w}_{t}=0
$$

Let us define the matrices $A=(1 / 2)\left(\Delta+\Delta^{\prime}\right)$ and $B=(1 / 2)\left(\Delta-\Delta^{\prime}\right) . A$ is a symmetric matrix whereas $B$ is a skew matrix. We have the following useful lemma:

Lemma 1 Let $\alpha_{1}$ and $\alpha_{2}$ the real eigenvalues of the matrix $\left(\beta^{1 / 2} \Gamma\right)^{-1}(-A)$ and $t_{1}$ and $t_{2}$ the associated eigenvectors. The matrix $T=\left[\begin{array}{ll}t_{1} & t_{2}\end{array}\right]$ can be choosen such that $T^{\prime}\left(-\beta^{1 / 2} \Gamma\right) T=I_{2}$ and $T^{\prime} A T=$ $\operatorname{diag}\left(\alpha_{1}, \alpha_{2}\right)$

The skew matrix $B$ implies:

$$
T^{\prime} B T=\left[\begin{array}{cc}
0 & d \\
-d & 0
\end{array}\right]
$$

We define $\widehat{w}_{t}=T \widehat{z}_{t}$ and (A.2) becomes :

$$
\left(T^{\prime} \Delta T\right) \widehat{z}_{t+2}+T^{\prime}\left(\beta^{1 / 2} \Gamma\right) T \widehat{z}_{t+1}+T^{\prime} \Delta^{\prime} T \widehat{z}_{t}=0
$$

From Lemma 1, we have:

$$
\left[\begin{array}{cc}
\alpha_{1} & d \\
-d & \alpha_{2}
\end{array}\right] \widehat{z}_{t+2}-\left[\begin{array}{cc}
1 & 0 \\
0 & 1
\end{array}\right] \widehat{z}_{t+1}+\left[\begin{array}{cc}
\alpha_{1} & -d \\
d & \alpha_{2}
\end{array}\right] \widehat{z}_{t}=0
$$

The parameters $\alpha_{1}, \alpha_{2}$ and $d$ are function of the structural parameters. The characteristic roots of equation (A.3) are solution of :

$$
\left(\alpha_{1} \alpha_{2}+d^{2}\right) \lambda^{4}-\left(\alpha_{1}+\alpha_{2}\right) \lambda^{3}+\left(2 \alpha_{1} \alpha_{2}+1-2 d^{2}\right) \lambda^{2}-\left(\alpha_{1}+\alpha_{2}\right) \lambda+\alpha_{1} \alpha_{2}+d^{2}=0
$$


This equation can be solved using $\mu=\lambda+\frac{1}{\lambda}$ and $\left(\alpha_{1} \alpha_{2}+d^{2}\right) \mu^{2}-\left(\alpha_{1}+\alpha_{2}\right) \mu+\left(1-4 d^{2}\right)=0$. Now consider the discriminant

$$
\kappa=\left(\alpha_{1}+\alpha_{2}\right)^{2}-4\left(1-4 d^{2}\right)\left(\alpha_{1} \alpha_{2}+d^{2}\right)
$$

In order to determine the roots of (A.3), one must consider two cases:

$$
\lambda_{j}+\frac{1}{\lambda_{j}}=\frac{\alpha_{1}+\alpha_{2} \pm \sqrt{\kappa}}{2\left(\alpha_{1} \alpha_{2}+d^{2}\right)} \text { if } \kappa>0 \text { and } \lambda_{j}+\frac{1}{\lambda_{j}}=\frac{\alpha_{1}+\alpha_{2} \pm i \sqrt{-\kappa}}{2\left(\alpha_{1} \alpha_{2}+d^{2}\right)} \text { if } \kappa<0
$$

for $j=1,2$. Note that the previous expressions define second order equations, whose coefficients are not necessarily real, i.e. the discriminant $\kappa$ can be negative. The eigenvalues of equation (A.1) are deduced using $\rho_{j}=\lambda_{j} / \sqrt{\beta}$.

Lemma 2 Let denote $\varphi_{1}=\left(1-4 d^{2}\right), \varphi_{2}=\left(\alpha_{1} \alpha_{2}+d^{2}\right), \varphi_{3}=\left(\alpha_{1}+\alpha_{2}\right), \bar{\beta}=\sqrt{\beta}+(1 / \sqrt{\beta})$ and $\beta=\sqrt{\beta}-(1 / \sqrt{\beta})$. Consider the dynamic system described by equation (A.1). The stationary equilibrium is a saddle path and its convergence path is (i) cyclical iff $4 \varphi_{1} \varphi_{2}>\varphi_{3}^{2}$ and $\left(\varphi_{1} / \varphi_{2}\right) \bar{\beta}>\left(\varphi_{3} / \varphi_{2}\right)^{2}+\bar{\beta}^{2} \underline{\beta}^{2}$ and (ii) monotone iff $4 \varphi_{1} \varphi_{2}<\varphi_{3}^{2},\left(\varphi_{3} / \varphi_{2}\right)>2 \bar{\beta}$ and $\bar{\beta}^{2}-\left(\varphi_{3} / \varphi_{2}\right) \bar{\beta}+\left(\varphi_{1} / \varphi_{2}\right)>0$.

Lemma 2 presents two types of convergence path toward the steady state. The first one is cyclical because the eigenvalues have no zero imaginary part. In the second case, the eigenvalues are real and the convergence is monotone. Lemma 2 presents only two cases. There exists also two other cases which are not discussed here: a case where the eigenvalues are both negative and a case where there exists both positive and negative eigenvalues. We will not discuss these two last cases, because negative eigenvalues cannot occur in our model.

\section{B Proof of proposition 1}

For $\alpha_{1}, \alpha_{2}$ and $d$, we have the following expressions :

$$
\begin{aligned}
\alpha_{1}+\alpha_{2} & =\frac{\beta^{1 / 2}}{\beta\left(\gamma_{11} \gamma_{22}-\gamma_{12}^{2}\right)}\left[\gamma_{12}\left(\delta_{12}+\delta_{21}\right)-\delta_{11} \gamma_{22}-\delta_{22} \gamma_{11}\right] \\
\alpha_{1} \alpha_{2} & ==\frac{\left[\delta_{11} \delta_{22}-1 / 4\left(\delta_{12}+\delta_{21}\right)^{2}\right]}{\beta\left(\gamma_{11} \gamma_{22}-\gamma_{12}^{2}\right)} \\
d^{2} & ==\frac{1 / 4\left(\delta_{12}-\delta_{21}\right)^{2}}{\beta\left(\gamma_{11} \gamma_{22}-\gamma_{12}^{2}\right)}
\end{aligned}
$$

From Lemma 2, we have a saddle path if the following inequalities are satisfied :

$$
\begin{array}{r}
\frac{\alpha_{1}+\alpha_{2}}{\alpha_{1} \alpha_{2}+d^{2}}>2(\sqrt{\beta}+1 / \sqrt{\beta}) \\
(\sqrt{\beta}+1 / \sqrt{\beta})^{2}-\frac{\alpha_{1}+\alpha_{2}}{\alpha_{1} \alpha_{2}+d^{2}}(\sqrt{\beta}+1 / \sqrt{\beta})+\frac{1-4 d^{2}}{\alpha_{1} \alpha_{2}+d^{2}}>0
\end{array}
$$

These two inequalities can be expressed with respect to the structural parameters:

$$
\frac{K^{* 2}}{\beta^{2}}\left[-\eta_{K}\left(-\frac{\phi s_{I}}{s_{C}} \xi_{C C}-\beta \frac{\alpha}{s_{C}}\left(\xi_{C C}-\xi_{L C}\right)+\frac{\alpha \beta}{\phi s_{I}} \phi \omega_{H H}\right)+\frac{\alpha \beta}{s_{C}} \frac{N}{1-N}\left(\xi_{C C} \xi_{L L}-\xi_{C L} \xi_{L C}\right)\right]>0
$$

and

$$
\frac{K^{* 2}}{\beta^{2}} \eta_{K}\left[(1-\beta) \frac{\alpha \beta}{s_{C}}\left(\xi_{C C}-\xi_{L C}\right)+\frac{\alpha \beta}{\phi s_{I}}\left(\frac{\alpha}{s_{C}}\left(\xi_{C C}-\xi_{L C}\right)+\frac{N}{1-N}\left(\xi_{L L}-\xi_{C L}\right)\right)\right]>0
$$

From the assumptions that consumption and leisure are normal goods and that $u($.$) is concave, we have$ $\xi_{C C}-\xi_{L C} \leq 0, \xi_{L L}-\xi_{C L} \leq 0$ and $\xi_{C C} \xi_{L L}-\xi_{C L} \xi_{L C}>0$. Moreover, $\eta_{K}<0$ and $\omega_{H H}>0$. It follows that the two inequalities are satisfied. This completes the proof. 


\section{Proof of proposition 2}

From proposition 1, the stationary equilibrium is a saddle path. To determine the nature of the adjustment path, we have to determine the sign of $\left(\alpha_{1}+\alpha_{2}\right)^{2}-4\left(1-4 d^{2}\right)\left(\alpha_{1} \alpha_{2}+d^{2}\right)$. From $\psi \omega_{H H}=\frac{b}{Z F_{N}} \equiv \frac{b}{W}$, the previous expression can be expressed as a second order polynomial in $b$

$$
f(b)=\frac{1}{W^{2}} \zeta_{1} b^{2}+\frac{1}{W} \zeta_{2} b+\zeta_{3}
$$

where

$$
\begin{aligned}
\zeta_{1} & =\beta\left(\frac{\alpha \beta}{\phi s_{I}}\right)^{2} \eta_{K}^{2} \\
\zeta_{2} & =\beta\left[4 \beta(1-\beta) \frac{\alpha}{s_{C}} \xi_{C C} \frac{\alpha}{s_{C}}\left(\xi_{C C}-\xi_{L C}\right) \eta_{K}-2 \frac{\alpha \beta}{\phi s_{I}} \frac{\alpha \beta}{s_{C}} \frac{N}{1-N}\left(\xi_{C C} \xi_{L L}-\xi_{C L} \xi_{L C}\right) \eta_{K}\right. \\
& +4 \frac{\alpha}{s_{C}} \xi_{C C} \frac{\alpha \beta^{2}}{\phi s_{I}}\left(\frac{\alpha}{s_{C}}\left(\xi_{C C}-\xi_{L C}\right)+\frac{N}{1-N}\left(\xi_{L L}-\xi_{C L}\right)\right) \eta_{K} \\
& \left.-2 \frac{\alpha \beta}{s_{I} \phi}\left(\beta \frac{\alpha}{s_{C}}\left(\xi_{C C}-\xi_{L C}\right)+\frac{\phi s_{I}}{s_{C}} \xi_{C C}\right) \eta_{K}^{2}\right] \\
\zeta_{3} & =\beta\left[\left(\beta \frac{\alpha}{s_{C}}\left(\xi_{C C}-\xi_{L C}\right)+\frac{\phi s_{I}}{s_{C}} \xi_{C C}\right) \eta_{K}+\frac{\alpha \beta}{s_{C}} \frac{N}{1-N}\left(\xi_{C C} \xi_{L L}-\xi_{C L} \xi_{L C}\right)\right]^{2}
\end{aligned}
$$

We now study the sign of this polynomial with respect to $b$. Without ambiguity, $\zeta_{1}>0$ and $\zeta_{3}>0$. If $\zeta_{2}<0$ and disc $=\zeta_{2}^{2}-4 \zeta_{1} \zeta_{3}>0$, the polynomial has two positive roots and it is negative if it is evaluated at values which lie between the two roots. The discriminant is given by $d i s c=T_{1} T_{2}$ with :

$$
\begin{aligned}
T_{1} & =\beta\left[4 \beta(1-\beta) \frac{\alpha}{s_{C}} \xi_{C C} \frac{\alpha}{s_{C}}\left(\xi_{C C}-\xi_{L C}\right) \eta_{K}\right. \\
& \left.+4 \frac{\alpha}{s_{C}} \xi_{C C} \frac{\alpha \beta^{2}}{\phi s_{I}}\left(\frac{\alpha}{s_{C}}\left(\xi_{C C}-\xi_{L C}\right)+\frac{N}{1-N}\left(\xi_{L L}-\xi_{C L}\right)\right) \eta_{K}\right] \\
T_{2} & =\left[\zeta_{2}-\beta\left(2 \frac{\alpha \beta}{\phi s_{I}} \frac{\alpha \beta}{s_{C}} \frac{N}{1-N}\left(\xi_{C C} \xi_{L L}-\xi_{C L} \xi_{L C}\right) \eta_{K}\right.\right. \\
& \left.\left.+2 \frac{\alpha \beta}{s_{I} \phi}\left(\frac{\alpha \beta}{s_{C}}\left(\xi_{C C}-\xi_{L C}\right)+\frac{\phi s_{I}}{s_{C}} \xi_{C C}\right) \eta_{K}^{2}\right)\right]
\end{aligned}
$$

$T_{1}$ is without ambiguity negative. We thus have to determine the sign of $T_{2}$. We introduce the following useful notations $\xi_{C C}-\xi_{L C}=-X$ and $\xi_{L L}-\xi_{C L}=-Y$. Therefore, $T_{2}$ becomes:

$$
T_{2}=4 \beta \frac{\alpha \beta}{s_{C}} \frac{\alpha \beta}{\phi s_{I}} \eta_{K}\left[\frac{\alpha}{s_{C}} X^{2}+\left(\eta_{K}-(1-\beta) \frac{\alpha}{s_{C}} \frac{\phi s_{I}}{\alpha \beta} \xi_{C C}\right) X+(1-\alpha) \xi_{C C}\right]
$$

Consider now the term in brackets :

$$
g(X)=\frac{\alpha}{s_{C}} X^{2}+\left(\eta_{K}-(1-\beta) \frac{\alpha}{s_{C}} \frac{\phi s_{I}}{\alpha \beta} \xi_{C C}\right) X+(1-\alpha) \xi_{C C}
$$

As $(1-\alpha) \xi_{C C}<0$ and $\frac{\alpha}{s_{C}}>0$, the above polynomial has a positive discriminant. The two roots have opposite sign. For values of $X$ greater than the positive root, the above expression is also positive. Consider now :

$$
g(1)=\alpha \beta(1-\delta)+\frac{(1-\alpha) \beta \alpha \delta}{1-\beta(1-\alpha \delta)}\left(1+\xi_{C C}\right)
$$

A sufficient condition for $g(1)$ be positive is $\xi_{C C} \geq-1$. Moreover, if $X=-\left(\xi_{C C}-\xi_{L C}\right) \geq 1$, then $T_{2}$ is negative and $\zeta_{2}$ is also necessarily negative. To sum up, we have disc $=\zeta_{2}^{2}-4 \zeta_{1} \zeta_{2}>0$ and $\zeta_{2}<0$ and equation (C.1) has two positive real roots. We conclude that there exists two positive real numbers $0<\underline{b}<\bar{b}<+\infty$ such that for all $b \in] \underline{b}, \bar{b}$, equation (C.1) is negative and complex eigenvalues occur. This completes the proof. 


\section{Proof of proposition 3}

The proof follows the one of C. Consider equation (C.2) and suppose that $\alpha>\delta$. We have :

$$
g(1)=\frac{\alpha \beta}{1-\beta(1-\alpha \delta)}\left[(1-\beta(1-\delta))(1-\alpha \delta)+(1-\alpha) \delta \xi_{C C}\right]
$$

It is then easy to verify that if $\alpha>\delta$, then $(1-\beta(1-\delta))(1-\alpha \delta)((1-\alpha) \delta))^{-1}>(1-\alpha \delta)(1-\alpha)^{-1}>1+\alpha$. The end of the proof is then similar to the one of proposition 2 . This completes the proof. 


\section{References}

Azariadis, C., J. Bullard, and L. Ohanian, Complex eigenvalues and trend-reverting fluctuations, mimeo, Federal Reserve bank of Saint-Louis 2001.

Burnside, C., M. Eichenbaum, and S. Rebelo, Labor hoarding and the business cycle, Journal of Political Economy, april 1993, 101 (2), 245-273.

Cassing, S. and T. Kollintzas, Recursive factor of production interrelations and endogenous cycling, International Economic Review, 1991, 32 (2), 417-440.

Cogley, T. and J.M. Nason, Output dynamics in real-business-cycle models, American Economic Review, June 1995, 85 (3), 492-511.

Fairise, X. and T. Langot, Labor productivity and the business cycle: can RBC be saved?, European Economic Review, october 1994, 38 (8), 1581-1594.

Hansen, G.D., Indivisible Labor and the Business Cycles, Journal of Monetary Economics, November 1985, 16 (3), 309-327.

Hercowitz, Z. and M. Sampson, Output growth, the real wage and employment fluctuations, American Economic Review, 1991, 81, 1215-1237.

King, R. and S. Rebelo, Resuscitating Real Business Cycles, in Handbook of Macroeconomics, J. Taylor and M. Woodford, 1999, chapter 14, pp. 927-1007.

Kydland, F. and E. Prescott, Time to build and aggregate fluctuations, Econometrica, 1982, 50, 1345-1370.

Magill, M., The stability of equilibrium, International Economic Review, 1979, 20, 577-597.

Oi, W., Labor as a quasi-fixed factor, Journal of Political Economy, 1962, 70.

Rogerson, R., Indivisible labor, lotteries and equilibrium, Journal of Monetary Economics, 1988, 21 (1), 3-17. 